\section{RESISTANCE TO ONCOLYTIC VACCINIA CAN BE REVERSED BY TARGETING REGULATORY T CELLS WITH VACCINIA-DIRECTED DELIVERY OF A TGF $\beta$ INHIBITOR}

${ }^{1}$ Kristin DePeaux*, ${ }^{1}$ Dayana Rivadeneira, ${ }^{1}$ McLane Watson, ${ }^{1}$ Andrew Hinck, ${ }^{2}$ Stephen Thorne, ${ }^{1}$ Greg Delgoffe. ${ }^{1}$ University of Pittsburgh, Pittsburgh, PA, United States; ${ }^{2}$ Kalivir Immunotherapeutics, Pittsburgh, PA, United States

Background Oncolytic viruses are an underappreciated immunotherapy capable of inflaming the tumor microenvironment (TME), vaccinating a patient against their own tumor, and delivering gene therapy to the TME. However, apart from the oncolytic HSV T-vec, these therapies have not seen widespread use, due in part to incomplete understanding of their immunologic mechanisms of action. We sought to determine features of oncolytic vaccinia virus (VV) response and resistance using subclones of the HPV+ head and neck cancer model MEER rendered sensitive or resistant to VV.

Methods A VV sensitive MEER tumor resisting treatment was serially passaged in mice and treated with VV until a stably resistant line was generated (Fig1). Sensitive or resistant MEER tumors were implanted, treated with a single intratumoral dose of VV, and harvested 4-7 days later for cytometric analysis. A genetically encoded TGF $\beta$ inhibitor was recombined into oncolytic VV (VV-TGFßi).

Results We used serial in vivo passaging to generate a VVresistant MEER line (MEERvvR) from one sensitive to VV (MEERvvS, figure 1) and compared their immune infiltrate. While VV promoted acute cytokine production and cytotoxicity in conventional $\mathrm{T}$ cells, the major determining factor between sensitivity and resistance was the phenotype of Treg cells. At baseline, Treg cells in MEERvvS had lower Nrp1 expression and higher IFN $\gamma$-STAT1 signaling compared to MEERvvR, indicative of Treg 'fragility'. VV treatment induced MEERvvS Treg cells to become immunostimulatory and produce IFN $\gamma$ (figure 2). RNAseq revealed MEERvvR produced more TGF $\beta$ than MEERvvS cells, suggesting these tumors directly stabilize Treg cells. To determine if MEERvvR could be sensitized to VV, we engineered oncolytic vaccinia to produce a genetically-encoded TGF $\beta$ inhibitor which binds TGF $\beta R I I$, preventing TGF $\beta 1-3$ binding (VV-TGF $\beta \mathrm{i}$ ). When MEERvvR were treated with VV-TGF $\beta$ i, elite responses were restored, with commensurate increase in survival (figure 3) associated with increased STAT1 signaling in Treg cells.

Conclusions Resistance to oncolytic vaccinia is controlled by Treg cell phenotype; tumors harboring more fragile Treg cells respond exquisitely to VV. An oncolytic vaccinia engineered to produce a novel TGF $\beta$ i could remodel the TME to be less supportive of Tregs, rendering resistant tumors sensitive to VV. Our data highlight the importance of Treg cell status in resistance to oncolytic virus therapy and suggest TGF $\beta$ can be effectively targeted through an inhibitor encoded within the virus. Importantly, this TME directed production of the TGF $\beta$ i carries no toxicity previously associated with systemic TGF $\beta$ inhibition, suggesting a viral approach to TGF $\beta$ inhibition can be an effective strategy support broader immunotherapy response.

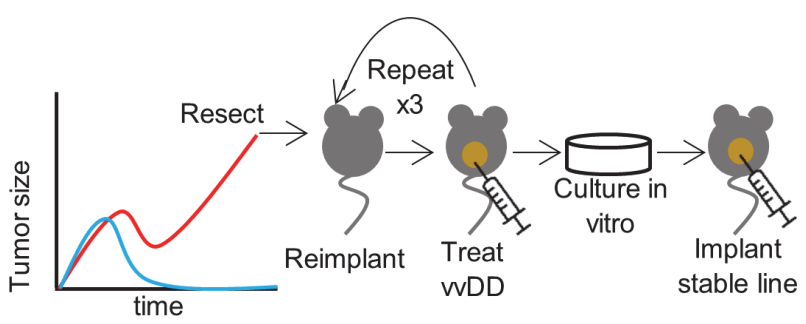

Abstract 743 Figure 1 Strategy used to generate a vaccinia resistant MEER (MEERvvR) from vaccinia sensitive MEER (MEERvvS)

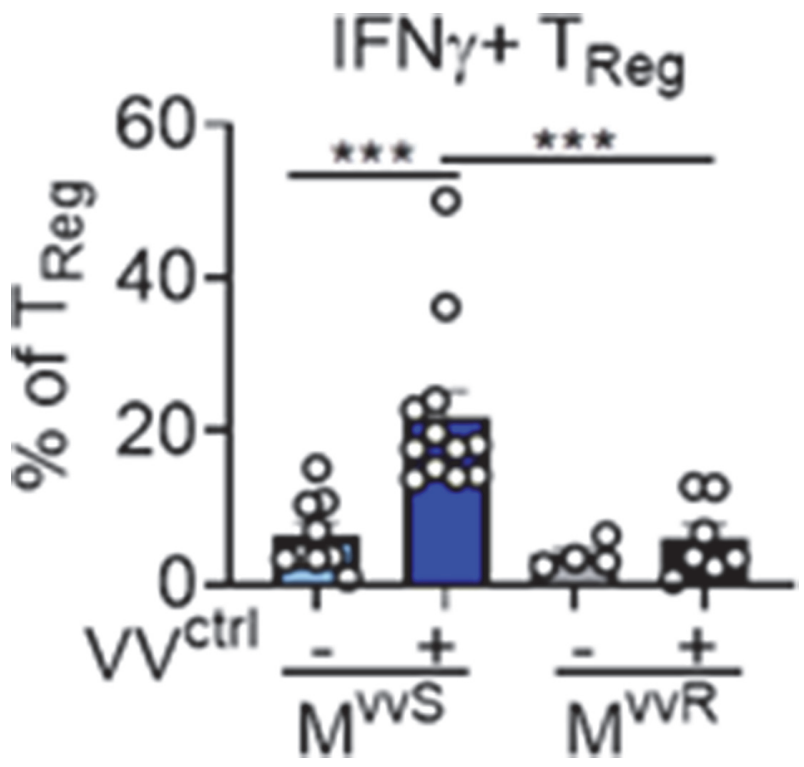

Abstract 743 Figure 2 IFN $\gamma$ production in Treg cells in MEERvvS and MEERvvR after treatment with PBS or control vaccinia (VV-Ctrl)

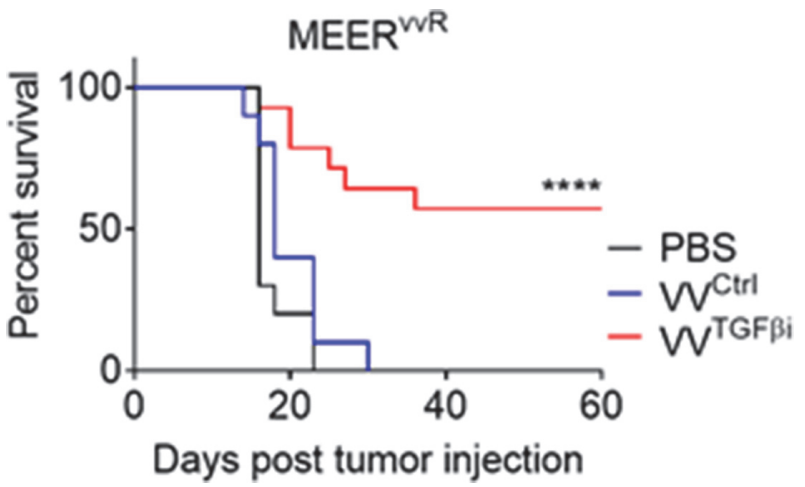

Abstract 743 Figure 3 Survival of VV-resistant MEER treated with $\mathrm{PBS}$, control vaccinia (VV-Ctrl), or vaccinia engineered to deliver a potent inhibitor of TGF $\beta$ (VV-TGF $\beta$ i)

http://dx.doi.org/10.1136/jitc-2021-SITC2021.743 